\title{
ESTUDIO DE LA CINÉTICA DE BIOSORCIÓN DE PLOMO (II) EN ALGA Ascophyllum Nodosum
}

\author{
Neptalí Ale Borja, ${ }^{a}$ Víctor García Villegas, ${ }^{a}$ Andrea Yipmantin Ojeda, ${ }^{b}$ Enrique Guzmán \\ Lezama, ${ }^{\mathrm{a}, \mathrm{b}}$ Holger Maldonado García. ${ }^{\mathrm{a}, \mathrm{b}}$
}

\begin{abstract}
RESUMEN
Se ha estudiado la influencia del pH y la cinética de biosorción de plomo a $20^{\circ} \mathrm{C}$ en el alga Ascophyllum nodosum. Para aumentar la estabilidad estructural del alginato e impedir su lixiviación, la biomasa fue pre-tratada con $\mathrm{CaC}_{12}$. Los datos experimentales de cinética de biosorción presentaron mayores coeficientes de correlación para el modelo matemático de pseudo segundo orden. Los parámetros fisicoquímicos: influencia del tamaño de partícula $(75 \mu \mathrm{m}-180 \mu \mathrm{m}$ y $0 \mu \mathrm{m}-75 \mu \mathrm{m})$, dosis de adsorbente y concentración de metal fueron evaluados en la cinética de biosorción. La constante de pseudo segundo orden, $k_{2}=1,0 \times 10^{-4}$ g. $\mathrm{mg}^{-1} \mathrm{~min}^{-1}$, para el biosorbente con tamaño de partícula de 75-180 $\mu$ m aumentó en 20\% cuando la granulometría disminuyó a 0-75 $\mu \mathrm{m}$, mientras que la capacidad de sorción en el equilibrio, $q_{2 e}$ se incrementó de 67,7 a $94,5 \mathrm{mg} / \mathrm{g}$ a las mismas condiciones. La concentración residual de plomo (II) en las soluciones se determinó mediante espectroscopía de absorción atómica. Para el procesamiento de datos y tratamiento estadístico se empleó el programa informático Origin 6.0, ajustándolos mediante regresión no lineal a los modelos de pseudo primer orden y pseudo segundo orden.

Palabras clave: Biosorción, Ascophyllum nodosum, plomo, cinética, pseudo-primer orden, pseudo-segundo orden
\end{abstract}

\section{KINETIC STUDY OF LEAD (II) BIOSORPTION ON Ascophyllum Nodosum SEAWEED}

\begin{abstract}
Biosorption optimal $\mathrm{pH}$ and kinetics of lead (II) on Ascophyllum nodosum seaweed at $20^{\circ} \mathrm{C}$ were studied. In order to improve structural stability of alginate and avoid leaching, the biomass was pre-treated with $\mathrm{CaCl}_{2}$. Biosorption kinetics experimental data showed grater coefficients of correlation $\left(\mathrm{R}^{2}\right)$ on pseudo second order mathematic model. Physical chemistry parameters: particle size influence $(75 \mu \mathrm{m}-180 \mu \mathrm{m}$ and $0 \mu \mathrm{m}-75 \mu \mathrm{m})$ and biosorbent and metal concentrations in solution were evaluated in the biosorption kinetics. Pseudo second order constant, $k_{2}=1.0 \times 10^{-4} \mathrm{~g} \cdot \mathrm{mg}^{-1} \mathrm{~min}^{-1}$, of biosorbent with particle size $75-180 \mu \mathrm{m}$ increased in $20 \%$ as particle size decreased to $0-75 \mu \mathrm{m}$, meanwhile, $q 2_{e}$ increased from 67,7 to 94,5 $\mathrm{mg} / \mathrm{g}$ at the same conditions. Lead (II) residual concentrations in solution were determined by atomic absorption spectroscopy. For data processing and statistic treatment of non-linear regression, Origin 6.0 informatics program was used.

Key words: Biosorption, Ascophyllum nodosum, lead, kinetic, pseudo-first order, pseudosecond order.

${ }^{a}$ Facultad de Química e Ing. Quimica., Universidad Nacional Mayor de San Marcos, Lima 1 nalebunmsm@gmail. com

${ }^{\mathrm{b}}$ Facultad de Ciencias y Filosofía Alberto Cazorla Talleri. Departamento de Ciencias Exactas-Sección Química, Universidad Peruana Cayetano Heredia, Lima 31




\section{INTRODUCCIÓN}

El incremento en la actividad industrial ha intensificado la contaminación ambiental y el deterioro de algunos ecosistemas, con la acumulación de agentes contaminantes, tales como metales pesados, compuestos sintéticos, líquidos de desechos nucleares, entre otros. En la actualidad, se está mostrando creciente atención al potencial peligro a la salud que representa la presencia de metales pesados en el ambiente. Por ello, los efluentes de desecho provenientes de la industria minera y metalúrgica son considerados como las mayores fuentes de contaminación por metales pesados y la necesidad de métodos económicos y efectivos para la remoción de estos contaminantes, ha resultado en el desarrollo de nuevas tecnologías de separación. ${ }^{1}$

Algunas actividades económicas generan la movilización de especies metálicas, las cuales son descargadas en el medio ambiente y tienden a persistir indefinidamente, acumulándose eventualmente a lo largo de la cadena alimenticia, creando así un serio problema al ecosistema. Es esencial comprender que el metal es solamente "removido" de una solución cuando es apropiadamente inmovilizado. El procedimiento de remoción de metales de soluciones acuosas a menudo orienta a una concentración efectiva del metal. A partir del proceso natural de mineralización del metal, que es bastante lento, la remoción final se alcanza solamente cuando el metal se convierte en concentrado al punto que puede retornar al proceso o ser aprovechado comercialmente. Este aspecto de la operación está dirigido a la recuperación potencial del metal, el cual, idealmente, debería ir mano a mano con los aspectos de remoción, haciendo que los procesos abarquen finalmente procedimientos efectivos para controlar la utilización de metales por humanos en sus procesos tecnológicos. ${ }^{2,3}$

Además, a causa de fuentes dispersas de disolución y/o lixiviación de metales, grandes proporciones de especies de metales pesados son lanzadas o descargados en el ambiente a partir de aguas de desecho industrial, como parte de la estructura ineficiente en las actividades tecnológicas usadas directamente en el procesamiento de metales. ${ }^{2-4}$

Con el fin de proponer alternativas de eliminación para los contaminantes, se han llevado a cabo un sinnúmero de esfuerzos, entre ellos el desarrollo de técnicas de precipitación, intercambio iónico, procesos electroquímicos y/o procesos de membrana: osmosis reversa, filtración; los cuales son comúnmente aplicados en el tratamiento de efluentes industriales. Sin embargo, la aplicación de tales técnicas es algunas veces restringido a causa del costo económico de la técnica. Por ello, dichos métodos no son efectivos ni aplicables a bajas concentraciones de contaminantes en disolución, tornándose altamente costosos y de bajo rendimiento a condiciones reales; como por ejemplo, la precipitación química y tratamiento electroquímico son ineficientes para la eliminación de metales pesados, especialmente cuando la concentración del metal en disolución acuosa es alrededor de $100 \mathrm{mg} / \mathrm{L}$; además, producen una elevada cantidad de lodos, cuyo tratamiento es de por sí difícil y costoso. Las resinas de intercambio iónico, adsorción por carbón activo y tecnología de membranas, son procesos extremadamente caros, especialmente cuando se tratan grandes volúmenes de efluentes de desechos conteniendo mezclas de metales pesados y especies orgánicas en bajas concentraciones, lo que los hace poco recomendables para su aplicación a gran escala. ${ }^{5-8}$ 
La investigación de nuevas tecnologías que involucran la remoción de metales tóxicos de aguas de desecho ha centrado su atención en los procesos de biosorción. Este término describe la remoción de metales pesados por un enlazamiento pasivo a biomasa no viva, a partir de soluciones acuosas. Esto implica que el mecanismo de remoción no es metabólicamente controlado.

En contraste, el término bioacumulación describe un proceso activo por el cual la remoción de metales requiere la actividad metabólica de organismos vivos.

La biosorción de metales pesados en soluciones acuosas puede ser considerada como una alternativa tecnológica en el tratamiento de aguas de desecho industrial. Estos procesos de innovación en tecnología de separación usan biomateriales, los cuales son abundantes, tal como algas marinas, desechos provenientes de la producción industrial, procesos biológicos y actividades derivadas de la agricultura.

El alga Ascophyllum nodosum es una fuente significativa de alginatos, el cual es un polisacárido que tiene igual valor comercial que el agar. Esta especie es usada como indicador biológico de polución por metales pesados a causa de la precipitación de sus alginatos por metales divalentes.2-9 Esta alga procede de las costas del mar atlántico de Francia.

\section{Capacidad de adsorción}

La calidad del material adsorbente es considerada de acuerdo a cuánto sorbato puede atraer y retener en una forma "inmovilizada". La cantidad de metal adsorbido por el adsorbente puede ser calculada como la capacidad de sorción, q. Las capacidades de sorción dependen de las constantes ácido-básicas, del punto isoeléctrico del adsorbente y del pH de la solución. ${ }^{7}$

Donde:

$$
q=\frac{V\left(C_{0}-C_{e q}\right)}{m}
$$

q: Capacidad de adsorción (mg.g $\left.\mathrm{g}^{-1}\right)$

$V$ : Volumen de la solución (L)

$C_{0}$ : Concentración inicial de plomo (ppm)

$C_{e q}$ : Concentración del residual de plomo en el equilibrio (ppm)

$m$ : Masa del biosorbente (g)

\section{Modelo cinético de pseudo primer orden (Lagergren, 1898)}

Este modelo asume la biosorción como una reacción de primer orden en cada uno de sus reactantes a partir de:

$$
\frac{d q_{t}}{d t}=k_{1}\left(q_{e}-q_{t}\right)
$$

Al resolver la ecuación diferencial tomando en cuenta los límites de integración desde $\mathrm{t}=0$ hasta $\mathrm{t}=\mathrm{t}$ se tiene:

$$
q_{t}=q_{e}\left(1-e^{-k_{1} t}\right)
$$


Donde:

$q_{e}$ : Cantidad de soluto adsorbido en el equilibrio por unidad de masa de adsorbente, $\left(\mathrm{mg} \cdot \mathrm{g}^{-1}\right)$.

$q_{t}$ : Cantidad de soluto adsorbido en el equilibrio por unidad de masa de adsorbente al tiempo $t$, (mg. $\left.\mathrm{g}^{-1}\right)$.

$k_{1}$ : Constante de velocidad de pseudo primer orden, $\left(\min ^{-1}\right)$.

La constante $k_{1}$ se puede determinar por análisis de regresión no lineal de la gráfica: $q_{t}$ vs $t$.

\section{Modelo cinético de pseudo segundo orden}

En este caso, la ecuación de velocidad de la cinética de adsorción se expresa como:

$$
\frac{d q_{t}}{d t}=k_{2}\left(q_{e}-q_{t}\right)^{2}
$$

Al resolver la ecuación diferencial tomando en cuenta los límites de integración desde $\mathrm{t}=0$ hasta $\mathrm{t}=\mathrm{t}$ se obtiene:

$$
q_{t}=\frac{q_{e^{2} k t}}{1+q_{e} k t}
$$

Donde:

$k_{2}$ : Constante de velocidad de pseudo segundo orden, $\left(\mathrm{g} \cdot \mathrm{mg}^{-1} \cdot \mathrm{min}^{-1}\right)$.

Las constantes $k_{2}$ y $q_{e q}$ se pueden determinar por análisis de regresión no lineal de la gráfica: $q_{t}$ vs $t$.

El objetivo de este trabajo fue encontrar el pH óptimo y el modelo matemático que represente mejor la cinética de biosorción de plomo en alga Ascophyllum nodosum.

\section{PARTE EXPERIMENTAL}

En esta investigación se utilizó el sistema batch (discontinuo), el cual consiste en colocar un volumen determinado de la solución del metal en un frasco o Erlenmeyer, agregar un peso determinado del sólido adsorbente y colocarlo en un agitador durante un tiempo suficiente para que estén en contacto el adsorbente y los iones metálicos presentes en la solución. Luego se filtra y determina en la solución filtrada la cantidad de metal no adsorbida en el sólido adsorbente.

\section{Obtención del alga:}

El Ascophyllum nodosum fue proporcionado por el Dr. Eric Guibal del laboratorio de investigación de Ing. Industrial y Medio Ambiente de la Escuela de Minas de Alés-Francia.

\section{Pretratamiento del alga}

$\mathrm{El}$ alga fue lavada con agua desionizada y secada en una estufa de vacío a $45^{\circ} \mathrm{C}$. Luego se molió y tamizó para obtener diferentes tamaños de partículas $(\mathrm{G}<5 \mu \mathrm{m}$ y $75 \mu \mathrm{m}<\mathrm{G}<180 \mu \mathrm{m})$; el tamizado se suspendió en una solución de $\mathrm{HCl} 0,1 \mathrm{~N}$ y se puso en agitación magnética constante (LAB-LINE) por una hora con el objeto de protonar los sitios activos. Se filtró al vacío y lavó con abundante agua desionizada para eliminar el exceso de ácido. Se dejó secar 
durante $24 \mathrm{~h}$ a temperatura ambiente. Finalmente, se refrigeró en frascos herméticos hasta su uso.

\section{Estabilización de Ascophyllum nodosum}

Este proceso se realizó mezclando $20 \mathrm{~g}$ de biomasa seca con $100 \mathrm{~mL}$ de solución de $\mathrm{CaCl}_{2} 0,2$ $\mathrm{M}$ a pH 5, ajustándolo con $\mathrm{NaOH} 1 \mathrm{M}$ y $\mathrm{HCl} 1 \mathrm{M}$ y en agitación durante $24 \mathrm{~h}$. Al término del tratamiento la biomasa fue lavada con abundante agua purificada por medio de un sistema de ósmosis reversa para remover cualquier exceso de $\mathrm{CaCl}_{2}$. El filtrado fue descartado y el alga reticulada fue secada a $45^{\circ} \mathrm{C}$ en una estufa al vacío durante $24 \mathrm{~h}$.

\section{Obtención del pH óptimo para la biosorción de $\mathbf{P b}^{2+}$}

Se preparó soluciones de 100 ppm de $\mathrm{Pb}^{+2}$ empleando $\mathrm{Pb}(\mathrm{NO} 3)_{2}$ y se vertió en 10 frascos de vidrio hasta un volumen de $200 \mathrm{~mL}$, ajustando cada muestra a diferentes $\mathrm{pH}(1,0 ; 1,9 ; 2,7$; 3,1 y 3,8 ). Se añadió $40 \mathrm{mg}$ de biosorbente (alga A. nodosum, $0-75 \mu \mathrm{m}$ ) a cada muestra y se colocó, seguidamente en el agitador orbital (Bigbill Digital Thermolyne) a $250 \mathrm{rpm}$, durante $72 \mathrm{~h}$ y a temperatura ambiente. Una vez transcurrido el tiempo, el biosorbente fue separado de las muestras; se filtró y se midió el $\mathrm{pH}$ final de las soluciones. La concentración residual de plomo, $c_{e}$ de la fase acuosa se determinó por espectrometría de absorción atómica.

\section{Cinética de biosorción de $\mathrm{Pb}$ (II)}

Para el estudio cinético, considerando el tamaño de partícula, se preparó 2 soluciones de 1 $\mathrm{L}$ de $\mathrm{Pb}$ (II) de 24,9 ppm ajustando al pH óptimo ( $\mathrm{pH}$ 4), se agregó $150 \mathrm{mg}$ de biosorbente $(0-75 \mu \mathrm{m} / \mathrm{L}$ y $75-180 \mu \mathrm{m} / \mathrm{L}$ en cada caso) y se puso en agitación magnética durante $48 \mathrm{~h}$ La clasificación del biosorbente, de acuerdo al diámetro de partícula, se obtuvo mediante el método de molienda y tamizado. Seguidamente se procedió a registrar el tiempo de reacción, considerando $t=0$ el instante en que el biosorbente se pone en contacto con la solución. A partir de $t=0$ se tomó muestras de $10 \mathrm{~mL}$ periódicamente hasta las $48 \mathrm{~h}$. Finalmente, se determinó la concentración de plomo $(c t)$ para cada muestra mediante la técnica de Espectrometría de absorción atómica de llama (equipo modelo Perkin Elmer AAnalyst 100).

Para el estudio de la cinética con diferentes concentraciones de metal se preparó tres soluciones de $1 \mathrm{~L}$ de sal de $\mathrm{Pb}$ (II) de 19,85; 49,96 y 199,45 ppm ajustando al pH óptimo ( $\mathrm{pH} 4)$ y luego se agregó $150 \mathrm{mg}$ de biosorbente $(0-75 \mu \mathrm{m} / \mathrm{L})$ a cada solución. La cinética de biosorción fue determinada para cada sistema de la misma forma que se describe en el análisis cinético considerando diferente tamaño de partícula.

En el estudio cinético con diferentes cantidades de biosorbente se preparó dos soluciones de $1 \mathrm{~L}$ de sal de plomo (II) de 24,9 ppm ajustando al pH óptimo (pH 4) y se agregó 100 y 200 $\mathrm{mg}$ de biosorbente $(0-75 \mu \mathrm{m} / \mathrm{L})$ a cada vaso, respectivamente. La cinética de biosorción fue determinada de la misma forma que se describe en los dos casos anteriores.

\section{Estabilización de Ascophyllum nodosum}

\section{RESULTADOS Y DISCUSIÓN}

Es un proceso que consiste en hacer reaccionar químicamente el material de desecho biológico 
o biopolímero para mantener su estructura y estabilidad y hacerlos resistentes a $\mathrm{pH}$ ácidos y así se evita la lixiviación de la materia orgánica, que consiste en que un disolvente líquido que pasa a través de un sólido pulverizado produce la elución de uno o más componentes del sólido. Este fenómeno de disolución implica la formación de un ion complejo, en el caso del Ascophyllum nodosum el alginato se solubiliza en el medio debido a que el agua es un agente de lixiviación que afecta la estructura y estabilidad del biopolímero y además, afecta las propiedades de adsorción de metales. ${ }^{10,11}$

Durante el proceso de reticulación se producen dos fenómenos químicos importantes: Primero se produce la protonación de la biomasa con $\mathrm{HCl}$; de esta forma el protón desplaza a los iones de los metales: $\mathrm{K}^{+}, \mathrm{Na}^{+}, \mathrm{Ca}^{2+} \mathrm{y} \mathrm{Mg}^{2+}$ adsorbidos por los grupos funcionales (ácido carboxílico, ácido sulfónico y otros) del alga. En segundo lugar, se hace reaccionar la biomasa con una solución acuosa de un ion dado en alta concentración $\left(\mathrm{Ca}^{2+}\right)$ para que este ocupe la mayoría de grupos funcionales cercanos.

La capacidad de los grupos alginatos de la superficie de las algas de intercambiar iones $\mathrm{Ca}^{2+}$ por medio de la liberación de iones $\mathrm{Na}^{+}, \mathrm{K}^{+}$e $\mathrm{H}_{3} \mathrm{O}^{+}$permite mejorar sus propiedades físicas y mecánicas mediante el entrecruzamiento de polialginato y formar la estructura denominada Caja de Huevos (figura 1) y de este modo se otorga una mayor estabilidad al alginato y favorecer el intercambio con otros iones de metales divalentes ${ }^{12}$.

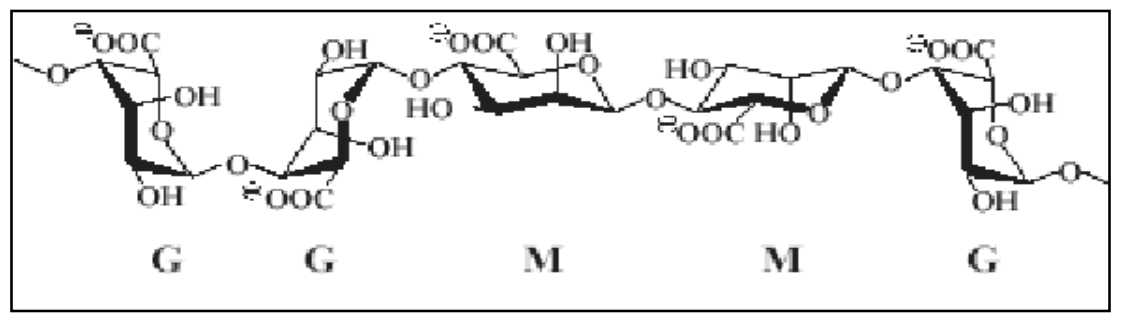

Figura 1. Polímero de alginato. Formación de gel vía bloques G, el "modelo caja de huevos"

\section{Influencia del pH}

El pH en el cual se produce la mayor sorción se debe a que la cantidad de grupos carboxilos presentes en forma aniónica es la mayor posible. En la figura 2 se observa que este valor ( $\mathrm{q}=234,5 \mathrm{mg} / \mathrm{g}$ ) se encuentra en el rango de $\mathrm{pH} 4-5,5$. Estos valores son similares a los obtenidos por Romera et al. (2006), citado por Wang et al. ${ }^{10}$ y también son semejantes a los que presentan otras algas pardas como Sargassum sp y Padina sp. ${ }^{11}$ La quelación con el metal debido a la desprotonación de los grupos carboxílicos de las unidades de alginato genera una disminución del $\mathrm{pH}$ con respecto al $\mathrm{pH}$ inicial. Sin embargo, esta disminución es menos notoria a valores más bajos de $\mathrm{pH}$ inicial debido a que un exceso de iones $\mathrm{H}^{+}$en la solución desplaza el equilibrio hacia la forma protonada del grupo carboxilo. 


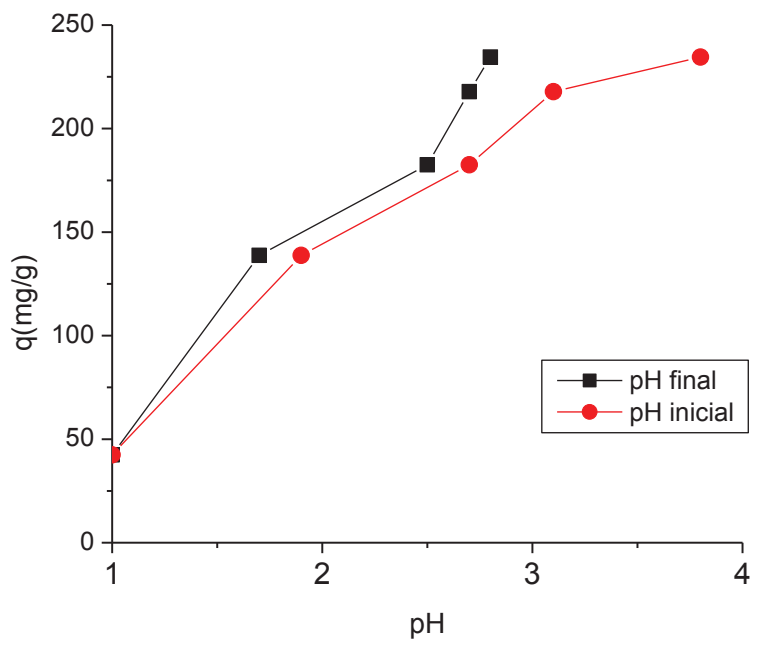

Figura 2. : Capacidad máxima de sorción, $q$, en $\mathrm{mg} / \mathrm{g}$ a diferentes valores de $\mathrm{pH}$ al inicio de la reacción y al final. Co: 100ppm, S: 40mg y V: $200 \mathrm{~mL}$

\section{Cinética de adsorción}

Los datos experimentales de la cinética de biosorción fueron ajustados a los modelos de pseudo primer orden y pseudo segundo orden. Estas curvas cinéticas fueron establecidas a partir de una solución de plomo (II) de concentración inicial conocida variando parámetros como dosis de biosorbente, tamaño de partícula de biosorbente y concentración inicial del adsorbato. Los parámetros cinéticos obtenidos por la aplicación de los modelos de pseudo primer orden y pseudo segundo orden, tales como las constantes cinéticas $k_{1}$ y $k_{2}$, el coeficiente de correlación $\mathrm{R}^{2}$ y las capacidades de adsorción de plomo (II) en el equilibrio $q_{e 1}$ y $q_{e 2}$, se encuentran en las tablas 1 y 2 . De acuerdo a nuestros resultados, se obtuvo una remoción de cerca del $90 \%$ del total de iones plomo (II) de la solución en los primeros 500 min para soluciones con una concentración del metal menor a 25 ppm. Sin embargo, en los sistemas con soluciones que presentaron concentraciones iniciales iguales o mayores a $50 \mathrm{ppm}$ se obtuvo el $90 \%$ de la remoción de iones plomo (II) en los primeros $120 \mathrm{~min}$. Los tiempos de remoción al 90\% fueron mayores a los obtenidos por Sheng et al. ${ }^{11}$ en el estudio de adsorción de plomo y otros metales pesados con las algas pardas: Sargassum sp. y Padina sp., el alga verde: Ulva sp, y el alga roja Gracillaria en donde se empleó mayores concentraciones de metal y biosorbente. Esto se debe, probablemente, a que el porcentaje de sitios activos y la desprotonación de grupos carboxilos aumentan con la disminución de la cantidad de biosorbente en la solución.

\section{Influencia del tamaño de partícula}

Muchos autores como $\mathrm{Aksu}^{13}$ señalan que la capacidad de adsorción disminuye en la mayoría de los casos al aumentar el tamaño de partícula debido a que las partículas más pequeñas presentan una mayor área superficial. En la figura 3 y la tabla 1 se puede observar dicha disminución para los sistemas S1 y S2. 
De acuerdo a estos resultados, si se aumenta el tamaño de partícula se producirá una disminución en la capacidad de adsorción, lo que indicaría que los grupos activos internos (que no forman parte de la superficie de la partícula) no son totalmente accesibles, por lo tanto la disminución del tamaño de las partículas permitía que algunos de estos grupos activos puedan estar presente para la unión con el metal.

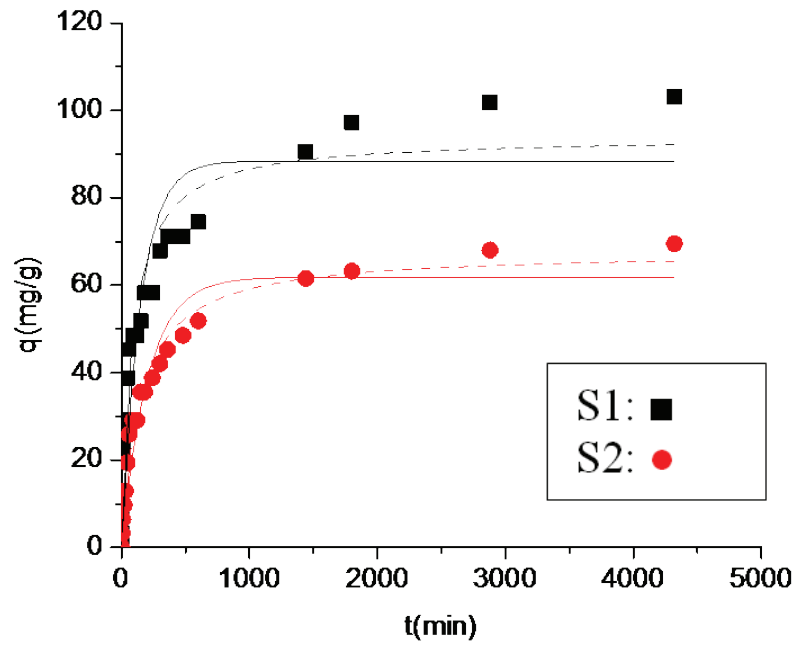

Figura 3. Curvas de tendencia de la cantidad de plomo adsorbida por unidad de masa de biosorbente con respecto al tiempo (qt en $\mathrm{mg} / \mathrm{g}$ ) para diferentes granulometrías de biosorbente $(0<\mathrm{S} 1<75 \mu \mathrm{m}<\mathrm{S} 2<180 \mu \mathrm{m})$ e igual concentración de metal y biosorbente (24,9 ppm de $\mathrm{Pb}$ y $150 \mathrm{mg}$ de alga). Los ajustes para el modelo de pseudo-primer orden están representados por la línea continua (—) y para el modelo de pseudo segundo orden por la línea punteada (- - - -).

El tamaño de partícula también influye en la pendiente inicial de las curvas. Si se disminuye el diámetro de las partículas, aumentará la pendiente de la curva cinética hasta un valor constante. Esto indica que la etapa inicial de la adsorción es influenciada por el tamaño de partícula debido a que ofrecen una mayor área superficial al interactuar con los iones metálicos.

Los parámetros cinéticos $k_{1}$ y $k_{2}$ disminuyeron con el incremento del tamaño de partícula $(6,2$ $-4,8 \times 10^{-3} \min ^{-1}$ para $k_{1}$ y $1,2-1,0 \times 10^{-4} \mathrm{~g} \cdot \mathrm{mg}^{-1} \cdot \mathrm{min}^{-1}$ para $\mathrm{k}_{2}$ ). Los valores de $k_{2}$ son similares a los obtenidos en la biosorción de plomo (II) en el alga parda Fucus vesiculosus ${ }^{14}$ y en el alga roja Chondracanthus chamissoi. ${ }^{15}$

\section{Influencia de la concentración del metal}

En la tabla 2 se observa que para concentraciones iniciales más diluidas de plomo (II) de 20 y $50 \mathrm{ppm}$ se tienen valores similares de $q_{e}(82,5$ y $89 \mathrm{mg} / \mathrm{g}$ en el modelo de pseudo primer y de 
104,4 y 98,1 mg/g para el modelo de pseudo segundo orden, respectivamente). Sin embargo, para una concentración inicial mayor del metal igual a 200ppm, la capacidad de adsorción en el equilibrio adquiere un valor máximo de $198,3 \mathrm{mg} / \mathrm{g}$ en el modelo de pseudo primer orden y de 102,6 mg/g para el modelo de pseudo segundo orden, respectivamente. Esto indica que en los sistemas S3 y S4 no se ha llegado aún a la saturación del biosorbente. También se observa que la concentración inicial del metal influye drásticamente en el valor de las constantes cinéticas $k_{1}$ y $k_{2}$.
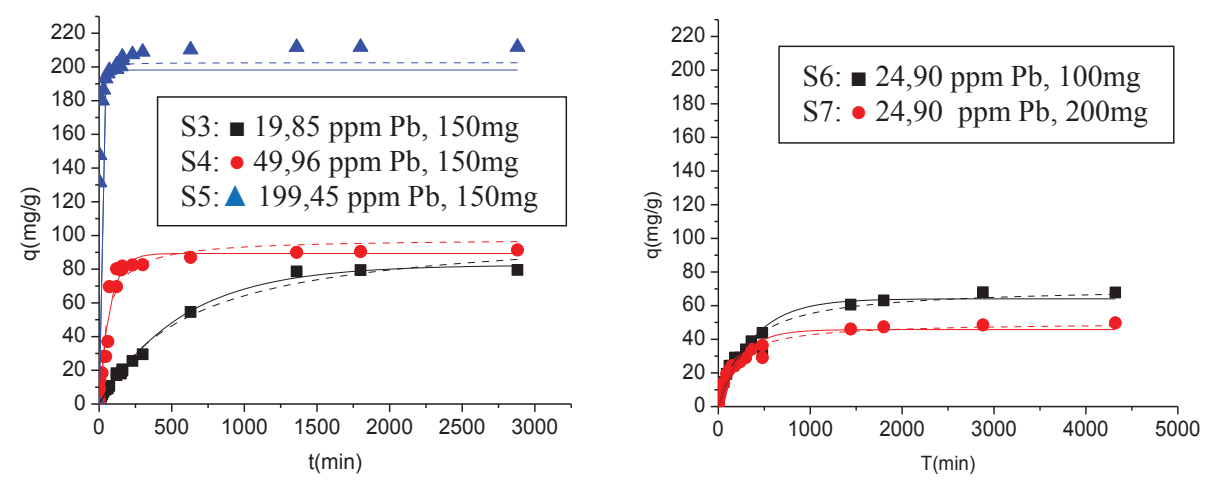

Figura 4. Curvas de tendencia de la cantidad de plomo adsorbido por unidad de masa de biosorbente con respecto al tiempo, $\mathrm{q}(\mathrm{t})$ en $\mathrm{mg} / \mathrm{g}$ para diferentes concentraciones de metal, manteniendo constante la masa de biosorbente (S3, S4 y S5) y para diferentes cantidades de biosorbente, manteniendo constante la concentración de metal (S6 y S7). Los ajustes para el modelo de pseudo-primer orden están representados por la línea continua (—) y para el modelo de pseudo segundo orden por la línea punteada (- - - - ).

\section{Influencia de la dosis de biosorbente}

En la tabla 2 se observa que al aumentar la dosis de biosorbente de 100 a $200 \mathrm{mg} / \mathrm{L}$, qe disminuye de 64,4 a 45,9 mg/g para el modelo de pseudo primer orden y de 71,7 a 50,0 mg/g para el modelo de pseudo segundo orden, debido a que se produce un cambio en la densidad de los sitios de adsorción. Con respecto a los parámetros de transferencia de masa, $\mathrm{k}_{1} \mathrm{y} \mathrm{k}_{2}$, se produjo un incremento de 2,9 a 4,0 x 10-3 min-1 para $\mathrm{k}_{1}$ y de 0,5 a 1,1 x 10-4 g.mg-1. min-1 para $\mathrm{k}_{2}$. La figura 4 muestra los perfiles cinéticos para $A$. nodosum bajo las condiciones experimentales ensayadas.

Tabla 1. Cinética de biosorción de plomo en alga Ascophyllum nodosum. Parámetros de las ecuaciones cinéticas de pseudo 1er orden y pseudo 2 do orden para diferentes tamaños de partícula (Ci $=24.9 \mathrm{ppm}$ y $\mathrm{S}=150 \mathrm{mg}$ )

\begin{tabular}{|c|c|c|c|c|c|c|}
\hline \multirow{2}{*}{$\begin{array}{c}\text { Tamaño de } \\
\text { partícula }\end{array}$} & \multicolumn{3}{|c|}{ Pseudo 1er orden } & \multicolumn{3}{|c|}{ Pseudo 2 do orden } \\
\hline & $\begin{array}{c}q_{e} \\
(\mathrm{mg} / \mathrm{g})\end{array}$ & $\begin{array}{l}k_{1} \times 10^{3} \\
\left(\mathrm{~min}^{-1}\right)\end{array}$ & $\mathbf{R}^{2}$ & $\begin{array}{c}q_{e} \\
(\mathrm{mg} / \mathrm{g})\end{array}$ & $\begin{array}{c}k_{2} \times 10^{5} \\
\left(\mathrm{~g} \cdot \mathrm{mg}^{-1} \mathrm{~min}^{-1}\right)\end{array}$ & $\mathbf{R}^{2}$ \\
\hline $\mathrm{S}^{*}{ }^{*}$ & 89,8 & 6,16 & 0,863 & 94,5 & 12 & 0,939 \\
\hline $\mathrm{S} 2{ }^{*}$ & 62,2 & 4,76 & 0,952 & 67,7 & 10 & 0,986 \\
\hline
\end{tabular}


Romera ${ }^{16}$ observó que cuanto más baja es la concentración de la biomasa en la suspensión, la relación metal/biosorbente será mayor y por lo tanto será más alta la cantidad de metal retenido por unidad de biosorbente hasta que la biomasa alcance la saturación. Si se aumenta la concentración de biomasa puede ocurrir que el exceso de ésta cubra los sitios activos (efecto cáscara) y por lo tanto se tendrá una disminución en la cantidad de metal adsorbido por unidad de biomasa. ${ }^{16}$

Tabla 2. Cinética de biosorción de plomo en alga Ascophyllum nodosum. Parámetros de las ecuaciones cinéticas de Pseudo 1er orden y Pseudo 2do orden (tamaño de partícula: 0 - $75 \mu \mathrm{m}$ ).

\begin{tabular}{|c|c|c|c|c|c|c|c|c|}
\hline \multirow{2}{*}{ Sistema } & \multirow{2}{*}{$\begin{array}{c}\mathrm{C}_{\mathrm{i}} \\
(\mathrm{ppm})\end{array}$} & \multirow{2}{*}{ m (mg) } & \multicolumn{3}{|c|}{ Pseudo 1er orden } & \multicolumn{3}{|c|}{ Pseudo 2 do orden } \\
\hline & & & $\begin{array}{c}q_{e} \\
(\mathrm{mg} / \mathrm{g})\end{array}$ & $\begin{array}{c}k_{1} \times 10^{-3} \\
\left(\min ^{-1}\right)\end{array}$ & $\mathbf{R}^{2}$ & $\begin{array}{c}q_{e} \\
(\mathrm{mg} / \mathrm{g})\end{array}$ & $\begin{array}{c}k_{2} \times 10^{-5} \\
\left(\mathrm{~g} \cdot \mathrm{mg}^{-1} \min ^{-1}\right)\end{array}$ & $\mathbf{R}^{2}$ \\
\hline $\mathrm{S} 3$ & 19,85 & 150 & 82,5 & 1,75 & 0,994 & 104,4 & 2 & 0,989 \\
\hline S4 & 49,96 & 150 & 89,3 & 13,63 & 0,967 & 98,08 & 19 & 0,951 \\
\hline S5 & 199,45 & 150 & $19, .3$ & 621,41 & 0,909 & 202,6 & 579 & 0,963 \\
\hline S6 & 24,9 & 100 & 64,4 & 2,95 & 0,962 & 71,7 & 5 & 0,979 \\
\hline S7 & 24,9 & 200 & 45,9 & 3,99 & 0,949 & 49,96 & 11 & 0,981 \\
\hline
\end{tabular}

\section{CONCLUSIONES}

La estereoquímica y presencia de grupos funcionales en los desechos de la biomasa determinan la formación de sitios activos responsables de la biosorción.

El tiempo de contacto entre el plomo (II) y el biosorbente para alcanzar las condiciones de equilibrio será grande (cerca de $500 \mathrm{~min}$ ) en sistemas en donde la concentración de metal sea baja (menor a $25 \mathrm{ppm}$ ), pero para concentraciones mayores a $50 \mathrm{ppm}$ se puede reducir hasta $120 \mathrm{~min}$. Como es de esperarse, la capacidad de adsorción se ve favorecida con la disminución del tamaño de partícula y con el incremento de la concentración inicial del metal.

\section{AGRADECIMIENTO}

Los autores agradecemos al Departamento de Ciencias Exactas-Sección Química de la Facultad de Ciencias y Filosofía Alberto Cazorla Talleri de la Universidad Peruana Cayetano Heredia y al Departamento de Química Analítica de la Facultad de Química e Ingeniería Química de la Universidad Nacional Mayor de San Marcos por el apoyo brindado en la presente investigación.

\section{BIBLIOGRAFÍA}

1. Viglio, F., Biolchini, F., Removal of Metales by biosorption: 2 review. Hidrometallurgy, 1997;44: 301-306.

2. Volesky, Bohumil, Sorption and Biosorption. Published by Bv Sorbex, Inc. - MontrealSt Lambert, Quebec, Canadá 2003. 
3. Volesky, B; Holan, ZR., Biosorption of heavy metals, Biotechnology Progress, 1995; 11(3): $235-250$.

4. Pagnanelli, F., Esposito, A., Toro, L., Viglio, F., Metal Speciation and $\mathrm{pH}$ effect on $\mathrm{Pb}$, $\mathrm{Cu}, \mathrm{Zn}$, and Cd biosorption onto Sphaeratilus natans: Langmuis-Type empirical model. Water Research, 2003; 37: 627-633.

5. Volesky, B: Biosorption for the next century, International Biohydrometallurgy Symposium, El Escorial, Spain, June 20 - 23. 1993.

6. Guibal, E., "Biosorción y adsorbentes para el tratamiento de efluentes minerometalúrgicos e industriales", Curso internacional, Universidad Peruana Cayetano Heredia, Facultad de Ciencias y Filosofía. 1997.

7. Tapia, N., “Aplicaciones ambientales de la biotecnología en la industria: Adsorción y Biosorción, Mecanismos y principales modelos para describir el fenómeno de biosorción”, Proyecto Palma, PUCP, 3, 5 y 6 de agosto, 1 - 17. 2002.

8. Kurniawan, Ton Agustiono; Chan, Gilbert Y.S.; Lo, Wai-Hung Y Babel, Sandhya. Physico-Chemical treatment techniques for wastewater laden with heavy metals Chemical Engineering Journal, 2006; 118 (1-2): 83-98.

9. Atkinson, BW, Bux F, Kasan HC., Biorremediación of Metal-Contaminated industrial effluents using waste sludges, Water Sci. Technol., 1996; 34: 9-15.

10. Wang, J. \& Chen, C., Biosorbents for heavy metals removal and their future. Biotechnology Advances, 2009; 27: 195-226.

11. Pin Xin Sheng, Yen-Peng Ting, J. Paul Chen and Liang Hong., Sorption of lead, cooper, cadmium, zinc, and nickel by marine algal biomass: Caracterization of biosorptive capacity and investigation of mecanisms, Journal of Colloid and Interface Science, 2004; 275: 131-141.

12. R. Jalali, H., Ghafourian, Y., Asef, SJ., Davarpanah, S., Sepehr, J. Haz. Mat., 2002; B92: 253-262.

13. Aksu, Z., Determination of the equilibrium, kinetic and thermodynamic parameters of the batch biosorption of nickel (II) ions onto Chlorella vulgaris. Process Biochemistry, 2002; 38: 89-99.

14. Mata, Y., Blázquez, M., Ballester, A. Gonzales, F. \& Muñoz, J., Characterization of the biosorption of cadmium, lead and copper with brown alga Fucus vesiculosus. Journal of Hazardous Materials, 2008; 158: 316-323. 
15. Yipmantin, A., Maldonado, J., Ly, M. Taulemesse, J \& Guibal, E., Pb(II) and Cd(II) biosorption on Chondracanthus chamissoi (a red alga). Journal of Hazardous Materials, 2011; 185: 922-929.

16. Romera, E., González, F., Ballester, A., Blázquez, M. L. \& Muñoz, J., Comparative study of biosorption of heavy metals using different types of algae, Bioresource Technology, 2007; 98: 3344-3353. 